\title{
Review: postmenopausal oestrogen replacement therapy increases the risk for venous thromboembolism
}

Miller J, Chan BK, Nelson HD. Postmenopausal estrogen replacement and risk for venous thromboembolism: a systematic review and meta-analysis for the U.S. Preventive Services Task Force. Ann Intern Med 2002 May 7;136:680-90.

\section{QUESTION: In postmenopausal women receiving hormone replacement therapy (HRT), what is the risk for venous thromboembolism (VTE)?}

\section{Data sources}

Studies were identified by searching Medline (1966 to 2000), HealthSTAR (1975 to 2000), and the Cochrane Controlled Trials Register, and by scanning the bibliographies of relevant studies and reviews.

\section{Study selection}

Studies were selected if they were published in English or had English-language abstracts, enrolled postmenopausal women, and reported deep venous thrombosis, pulmonary embolism, or VTE as adverse events related to oestrogen use. Studies that selected participants on the basis of previous thromboembolic events or risk for thrombosis were excluded.

\section{Data extraction}

Data were extracted on participants, treatment (for randomised controlled trials [RCTs]), definition and method for determining exposure (for case control and cohort studies), event rates, confounders, outcome measurement, and study duration. 2 reviewers independently rated study quality.

\section{Main results}

12 trials were included: 3 RCTs, 8 case control studies, and 1 cohort study. Risk for VTE was associated with use of HRT. The risk was evident in each of the 3 study types and when all 12 studies were meta-analysed (table). Among 6 studies that reported first year risk, the risk for a thromboembolic event was higher in the first year of HRT use than in subsequent years (relative risk [RR] 3.49, credible interval 2.3 to 5.6, $v$ RR 1.91, credible interval 1.2 to 3.5 ). The results were not appreciably affected when studies were grouped by quality rating or by presence or absence of coronary artery disease.

\section{Conclusion}

In postmenopausal women receiving hormone replacement therapy, risk for venous thromboembolism is twice that of women not receiving hormone replacement therapy.

Sources of funding: Agency for Healthcare Research and Quality; Portland Veterans Affairs Medical Center Women's Health Fellowship.

For correspondence: Dr J Miller, Oregon Health and Science University, Portland Oregon, USA. millerji@ohsu.edu.
Thromboembolic events in postmenopausal women receiving hormone replacement therapy (HRT) v no HRT*

\begin{tabular}{lll} 
Study type & $\begin{array}{l}\text { Number } \\
\text { of studies }\end{array}$ & $\begin{array}{l}\text { Relative risk (95\% } \\
\text { credible interval) } \dagger\end{array}$ \\
\hline Randomised controlled trial & 3 & $3.75(1.2$ to 10.3$)$ \\
\hline Cohort study & 1 & $2.10(1.2$ to 3.8$)$ \\
\hline Case control study & 8 & $2.05(1.4$ to 2.6$)$ \\
\hline All studies & 12 & $2.14(1.6$ to 2.8$)$ \\
\hline${ }^{*}$ A random effects model was used. \\
$\begin{array}{l}\text { †Credible interval is the range within which a } 95 \% \text { chance exists of finding the } \\
\text { true relative risk. }\end{array}$
\end{tabular}

\section{COMMENTARY}

The meta-analysis by Miller $e$ al and the Women's Health Initiative (WHI) study found almost identical estimates of risk for VTE in HRT users compared with those in non-users ${ }^{1}$ : RR 2.14 and 2.11, respectively. In the WHI study, however, HRT resulted in about 2 additional VTE events per 1000 users/year, which is a 10 -fold higher rate than that reported by Miller et al and others. ${ }^{2}$ The meta-analysis also found a consistent 2-fold to 3-fold greater risk for VTE in studies with different HRT preparations and in patients with various comorbid conditions, whereas the WHI investigated 1 HRT regimen in healthy women. This remarkable consistency of results, which was also seen across studies of different methodological rigour, probably reflects the robustness of the association between HRT and VTE and supports its generalisability to a broad spectrum of women and HRT preparations.

Miller $e$ al found that the risk for VTE is highest during the first year of HRT use (RR 3.49), which is consistent with the results from the WHI. The WHI also reported that a 1.5-fold to 2-fold risk increase persists for up to 5 years. One can speculate that VTE that occurs within months of beginning HRT may reflect the presence of an underlying thrombophilia, ${ }^{3}$ with HRT acting as a trigger for VTE, whereas VTE that occurs after several years of HRT reflects a more complex pathophysiology.

Several unresolved issues remain. First, the effect of progestogens on the risk for VTE was not addressed by the meta-analysis and cannot be inferred from the WHI until the findings from the oestrogen only treatment arm are available. Progestogens are not considered to have prothrombotic effects, and progestogen-only therapy is recommended for women with previous VTE. Second, the risk for VTE with raloxifene and transdermal HRT was not addressed in the meta-analysis. Emerging data suggest that these agents are associated with an increased risk for VTE and, until further data are available, should be avoided in women with previous VTE. Third, it is unclear whether HRT increases the risk for VTE in patients with thrombophilia (eg, factor V Leiden). Additional research may inform the role, if any, of thrombophilia screening before HRT is initiated.

The clinical implication of the meta-analysis is that HRT should be avoided in women with previous VTE, a group that had a 5-fold greater risk for recurrent disease in the WHI study (RR 4.90) ${ }^{1}$ and a previous study. ${ }^{4}$ However, because VTE is common and often presents in premenopausal women, treatment strategies are needed for the management of women with previous VTE and menopausal symptoms in whom HRT is the treatment of choice.

James Douketis, MD St Joseph's Hospital Hamilton, Ontario, Canada

1 Writing Group for the Women's Health Initiative Investigators. Risks and benefits of estrogen plus progestin in healthy postmenopausal

women. JAMA 2002;288:321-33. Hormone replacement therapy and risk of venous thromboembolism: population based case-control study. BMJ 1997;314:796-800.

Bloemenkamp KW, Rosendaal FR, Helmerhorst FM, et al. Higher risk of venous thrombosis during early use of oral contraceptives in wo

4 Høibraaten E, Qvigstad E, Arnesen H, et al. Increased risk of recurrent venous thromboembolism during hormone replacement therapy - results of the randomised, double-blind, placebocontrolled estrogen in venous thromboembolism trial (EVTET). Thromb Haemost 2000;84:961-7. 